\title{
Femtosecond solvation dynamics of indium dimers inside superfluid helium nanodroplets
}

\author{
Miriam Meyer ${ }^{1}$, Bernhard Thaler ${ }^{1}$, Pascal Heim ${ }^{1}$, and Markus Koch $^{1, *}$ \\ ${ }^{1}$ Graz University of Technology, Institute of Experimental Physics, $8010 \mathrm{Graz}$, Austria
}

\begin{abstract}
Indium dimers $\left(\operatorname{In}_{2}\right)$ solvated inside helium nanodroplets are studied with femtosecond photoelectron and photoion spectroscopy. The solvation dynamics triggered by photoexcitation of the $\operatorname{In}_{2}$ include expansion of the solvation shell and dopant ejection from the droplet, which both proceed more slowly than for In atoms. Oscillation of the solvation shell, in contrast, is similar to the case of In atoms. These processes are observed in combination with intrinsic dynamics of the molecule and set the time-frame for the dopant interaction with the quantum fluid environment.
\end{abstract}

\section{Introduction}

Superfluid helium nanodroplets $\left(\mathrm{He}_{\mathrm{N}}\right)$ represent a special approach for the synthesis of tailormade or fragile molecules and clusters. In view of time-resolved spectroscopy, they provide access to novel classes of systems that have eluded ultrafast dynamical studies so far. Pure $\mathrm{He}_{\mathrm{N}}$ [1] and surface-located alkali-metals [2] have been subject to femtosecond experiments. Concerning molecules immersed inside the $\mathrm{He}_{\mathrm{N}}$, the only attempt to observe ultrafast dynamics used the salt molecules sodium- and lithium iodide, which, however, was not successful [3].

Recently, we demonstrated that time-resolved photoelectron (PE) spectroscopy can be used to observe photoinduced dynamics of single atoms that are fully solvated inside the droplet [4]. The PE kinetic energy turned out to be a sensitive observable to follow the temporal evolution of the solvation shell around the photoexcited dopant. Supported by timedependent density functional theory, we developed the following mechanistic picture: Photoexcitation of a single indium (In) atom inside a $\mathrm{He}_{\mathrm{N}}$ leads to expansion of the solvation shell within $600 \mathrm{fs}$, followed by an oscillation of the bubble with a period of about $30 \mathrm{ps}$, and, finally, ejection of the In atom from the droplet after about $60 \mathrm{ps}$.

Here, we investigate the photoexcitation dynamics of the $\mathrm{In}_{2}-\mathrm{He}_{\mathrm{N}}$ system, for which we observe solvation dynamics similar to the monomer case, as well as intrinsic $\operatorname{In}_{2}$ molecular dynamics. In the presented work we concentrate on the former and interpret the corresponding transient signals by comparison to the previous $\mathrm{In}-\mathrm{He}_{\mathrm{N}}$ results.

\footnotetext{
${ }^{*}$ Corresponding author: markus.koch@tugraz.at
} 


\section{Method}

As described in detail previously [4, 5], He $\mathrm{N}_{\mathrm{N}}$ with a mean number of $\bar{N}=8000 \mathrm{He}$ atoms are generated in a supersonic expansion and doped inside a resistively heated pickup cell with two In atoms per droplet, which form an $\mathrm{In}_{2}$ molecule. Dynamics of the $\mathrm{In}_{2}-\mathrm{He}_{\mathrm{N}}$ system are investigated with a femtosecond pump-probe photoionization experiment with variable timedelay, where the kinetic energy of photoelectrons (PEs) and charge-to-mass ratio of ions are measured with a time-of-flight spectrometer. Pump pulses are obtained from a commercial Ti:sapphire laser system and frequency upconverted by an optical parametric amplifier to a photon energy of $3.59 \mathrm{eV}(345 \mathrm{~nm})$ in order to excite the $\mathrm{B}^{3} \Pi_{g}$ (II) $\leftarrow X^{3} \Pi_{u}$ transition of $\mathrm{In}_{2}$ inside $\mathrm{He}_{\mathrm{N}}$ [5]. Probe pulses are frequency doubled to $3.05 \mathrm{eV}(406 \mathrm{~nm})$.

\section{Results and Discussion}

Figure 1a shows the time evolution of the PE spectrum within the first two picoseconds after photoexcitation. The signal is modulated with a period of about $400 \mathrm{fs}$, which we ascribe to a nuclear wave packet oscillation of the $\mathrm{In}_{2}$ molecule. The periodic signal maxima correspond to ionization from the excited $\mathrm{B}^{3} \Pi_{g}$ (II) state to the cationic ground state of $\operatorname{In}_{2}$, at a certain nuclear distance of the two In atoms (Condon point) [6]. The $\mathrm{In}_{2}$ wave packet dynamics will be further analysed in an upcoming publication.

a

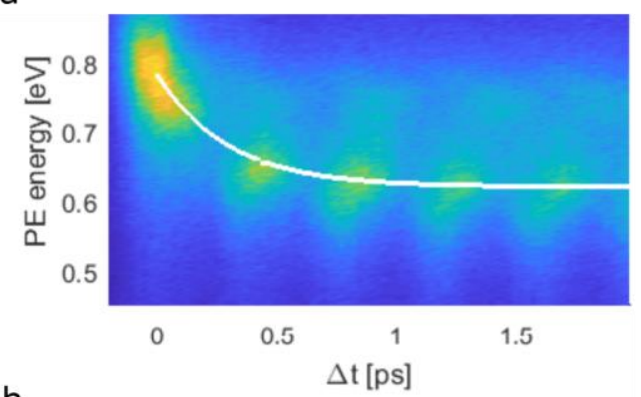

b

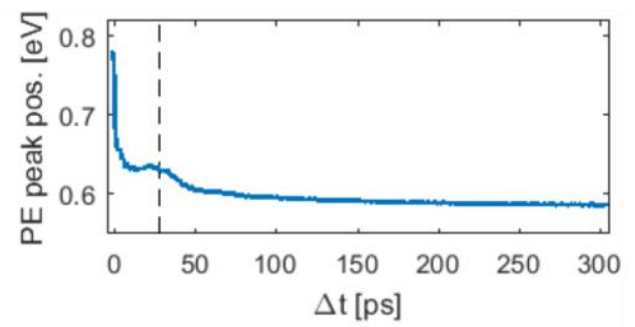

C

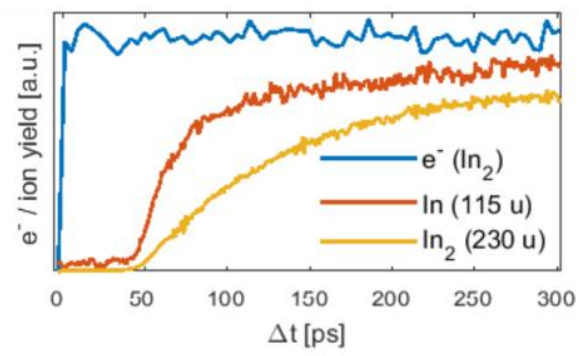

d

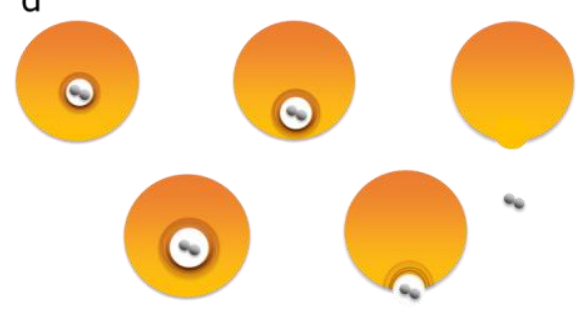

Figure 1: Time evolution of the $\mathrm{In}_{2}-\mathrm{He}_{\mathrm{N}}$ system after photoexcitation. (a) PE spectrum within the first 2 ps. The white line connects the signal maxima. (b) PE peak position up to $300 \mathrm{ps}$, as determined by Gaussian fits to the corresponding PE spectra. The dashed line at $28 \mathrm{ps}$ indicates the maximum of the temporal increase in PE energy. (c) Ion yields recorded at the In monomer/dimer masses of 115/230 u for photoexcitation at the monomer/dimer band at 376/345 nm (red/yellow line) and total PE yield integrated over all kinetic energies for excitation at $345 \mathrm{~nm}$ (blue line). (d) Sketch of the $\mathrm{In}_{2}$ ejection process.

The maxima are energetically shifted by $150 \mathrm{meV}$ from $780 \mathrm{meV}$ to $630 \mathrm{meV}$ within the first picosecond, as indicated by a white line in Figure 1a. This shift is related to the expansion of 
the He bubble as consequence of the enlargement of the valence electron orbital during photoexcitation [4]. Compared to the In-He system, where a shift of $290 \mathrm{meV}$ is observed within $1 \mathrm{ps}$ and $90 \%$ peak shift are completed within $500 \mathrm{fs}$, the shift of the $\mathrm{In}_{2}-\mathrm{He}_{\mathrm{N}}$ is smaller $(150 \mathrm{meV})$ and takes longer $(90 \%$ within $700 \mathrm{fs})$. Both indicate that the excited state interaction with the He solvation shell is less pronounced for the In dimer compared to the monomer.

The PE peak shift up to 300 ps is shown in Figure 1b. After a steep, initial decrease, representing the bubble expansion, the peak position is shifted further towards lower energies with a temporal energetic increase around 28 ps (marked by the dashed line), before it slowly approaches a constant value of $\sim 590 \mathrm{meV}$. The impulsive expansion of the solvation shell triggers an oscillation of the He bubble, the first contraction of which leads to the temporal increase in energy. This contraction is observed at the same time as for the monomer [4]. The decrease after 1 ps of the PE energy represents the ejection process of $\operatorname{In}_{2}$ from the droplet, as the ionization energy inside the $\mathrm{He}_{\mathrm{N}}$ is reduced due to polarization effects [7], compared to that of bare $\mathrm{In}_{2}$.

Ejection from the $\mathrm{He}_{\mathrm{N}}$ is particularly well observed by the transient ion yield. In Figure 1c the transient dimer ion yield for photoexcitation at the $\operatorname{In}_{2}$ band ( $345 \mathrm{~nm}$, yellow line) is compared to the monomer yield for excitation at the monomer band (376 nm, red line) [5]. Additionally, the total electron yield over time for excitation at $345 \mathrm{~nm}$ (blue line) is shown. While the electron signal shows an immediate rise at time-zero and remains constant afterwards, the ion signals both exhibit a delayed onset at $50 \mathrm{ps}$ followed by a faster rise of the monomer within $\sim 50$ ps and a slower dimer rise within $\sim 200$ ps. This shows that $\mathrm{In}_{2}$ is ejected from the droplet, in consequence of a repulsive, heliophobic excited state, as it is also the case for the In monomer [4]. Due to the cylindrical symmetry of $\operatorname{In}_{2}$ the repulsive force experienced by the molecule depends on its orientation inside the $\mathrm{He}_{\mathrm{N}}$, which is not the case for the spherically symmetric monomer. This would be a possible explanation for the similar onset time and slower rise of the dimer compared to the monomer.

In conclusion, the photoinduced solvation dynamics of the $\mathrm{In}_{2}-\mathrm{He}_{\mathrm{N}}$ system, as sketched in Figure 1d, show all three signatures previously observed for $\mathrm{In}-\mathrm{He}_{\mathrm{N}}$ [4] - bubble expansion, bubble oscillation and dopant ejection. Bubble expansion and ejection proceed more slowly and are less pronounced for $\mathrm{In}_{2}$ dopants, while the bubble oscillation is surprisingly similar to the atomic case.

We thank Wolfgang E. Ernst for providing the laser system and for fruitful discussions. We acknowledge financial support by the Austrian Science Fund (FWF) under Grant P29369-N36, as well as support from NAWI Graz.

\section{References}

1. M. P. Ziemkiewicz, D. M. Neumark, O. Gessner, Int. Rev. Phys. Chem. 34, 239 (2015)

2. M. Mudrich, F. Stienkemeier, Int. Rev. Phys. Chem. 33, 301 (2014)

3. H. Schmidt et al., J. Chem. Phys. 142, 44303 (2015)

4. B. Thaler et al., accepted by Nat. Comm., arXiv preprint arXiv:1804.04497 (2018)

5. B. Thaler, R. Meyer, P. Heim, S. Ranftl, J.V. Pototschnig, A.W. Hauser, M. Koch, W.E. Ernst, submitted (2018)

6. I. Fischer, M. J. J. Vrakking, D. M. Villeneuve, A. Stolow, Chem. Phys. 207, 331 (1996)

7. E. Loginov, D. Rossi, M. Drabbels, Phys. Rev. Lett. 95, 163401 (2005) 